\title{
Arthrogryposis-hyperkeratosis syndrome, lethal form
}

INSERM

\section{Source}

INSERM. (1999). Orphanet: an online rare disease and orphan drug data base.

Arthrogryposis-hyperkeratosis syndrome, lethal form. ORPHA:1485

Arthrogryposis-hyperkeratosis syndrome, lethal form is an arthrogryposis syndrome, described in two siblings to date, characterized by the association of multiple congenital joint contractures (of the large joints, fingers and toes) and hyperkeratosis (i.e. thick, scaling and fissured skin), with death occurring in early infancy. There have been no further reports in the literature since 1993. 